4-2018

\title{
Cognitive fusion and post-trauma functioning in veterans: Examining the mediating roles of emotion dysregulation
}

Daniel W. Cox

Thomas C. Motl

University of North Dakota, thomas.motl@UND.edu

A. Myfanwy Bakker

Rachael A. Lunt

How does access to this work benefit you? Let us know!

Follow this and additional works at: https://commons.und.edu/ehb-fac

Part of the Cognitive Psychology Commons

\section{Recommended Citation}

Daniel W. Cox, Thomas C. Motl, A. Myfanwy Bakker, et al.. "Cognitive fusion and post-trauma functioning in veterans: Examining the mediating roles of emotion dysregulation" (2018). Education, Health \& Behavior Studies Faculty Publications. 2.

https://commons.und.edu/ehb-fac/2

This Article is brought to you for free and open access by the Department of Education, Health \& Behavior Studies at UND Scholarly Commons. It has been accepted for inclusion in Education, Health \& Behavior Studies Faculty Publications by an authorized administrator of UND Scholarly Commons. For more information, please contact und.commons@library.und.edu. 
Cognitive fusion and post-trauma functioning in veterans: Examining the mediating roles of emotion dysregulation

Daniel W. Cox, University of British Columbia

Thomas C. Motl, University of British Columbia, University of North Dakota

A. Myfanwy Bakker, University of British Columbia

Rachael A. Lunt, Vancouver CBT Centre

Abstract: When cognitively fused, people have difficulty accepting and clearly perceiving their internal experiences. Following trauma, emotional non-acceptance and emotional non-clarity have been associated with post-trauma functioning. The aim of the present study was to integrate theory and research on cognitive fusion and posttrauma functioning to evaluate a theory-based model in which emotion dysregulation — specifically, emotional non-acceptance and emotional non-clarity — mediated the association between cognitive fusion and post-trauma functioning in a veteran sample. Participants were 149 veterans with a history of military-related trauma. Veterans completed measures of cognitive fusion, emotion dysregulation, posttraumatic stress disorder (PTSD) symptoms, and life satisfaction. Overall, emotion dysregulation and PTSD symptoms mediated the fusion-posttrauma functioning association in theoretically consistent ways. More specifically, fusion was related to PTSD through emotional non-clarity and fusion was related to goal dysregulation through emotional non-acceptance and PTSD. Our findings indicate that fusion impacts different aspects of post-trauma functioning through different mediators. How these different pathways could impact clinical decision making are discussed. Keywords: Cognitive fusion, Posttraumatic stress disorder, Military veteran, Emotion regulation, Life satisfaction, Acceptance and commitment therapy 
Trauma is an occupational hazard of military service. Veterans who experienced militaryrelated traumas tend to have more psychological dysfunction—including psychopathology and reduced life satisfaction — compared to those who did not (e.g., Surís, Lind, Kashner, \& Borman, 2007; Vogt, King, King, Saverese, \& Suvak, 2004). With improved understanding of what inhibits post-trauma functioning in this population, practices and services that aim to help veterans can be enhanced.

Cognitive fusion and emotion dysregulation have been linked with post-trauma functioning (e.g., Ehring \& Quack, 2010; Twohig, 2009; Walser \& Hayes, 2006). When people are cognitively fused, they are entangled in (i.e., fused with) their beliefs and hold those beliefs as literally true (Gillanders et al., 2014). This entanglement with thoughts reduces the ability to acknowledge and label thoughts and related emotions. Further, when thoughts are interpreted as truth, internal experiences are avoided rather than experienced. While theory has indicated that emotion dysregulation mediates the association between fusion and post-trauma functioning (Bardeen \& Fergus, 2016; Walser \& Hayes, 2006), no study has tested this association. Presently, we investigated a theoretically based model in which emotion dysregulationspecifically, emotional non-acceptance and emotional nonclarity — mediated the association between cognitive fusion and posttrauma functioning (e.g., posttraumatic stress disorder [PTSD] symptoms, goal dysregulation, life satisfaction) among veterans with military- related trauma.

\section{Cognitive fusion and PTSD}

Following traumatic events, beliefs about the self, the world, and the traumatic events are key predictors of the development and maintenance of PTSD (e.g., Foa, Ehlers, Clark, Tolin, \& Orsillo, 1999). Examples include believing that the self is totally incompetent, the world is utterly dangerous, and the trauma was the victim's fault. While the traditional cognitive- 
behavioral perspective is that these cognitions are maladaptive and interventions should target modifying them (Cahill, Rothbaum, Resick, \& Follette, 2009), the Acceptance and Commitment Therapy (ACT) perspective is that interventions should target defusing people from these beliefs rather than altering the beliefs themselves (Twohig, 2009; Walser \& Hayes, 2006). In the case of PTSD, defusing with beliefs about the world being dangerous and the self being incompetent facilitates the willingness to experience internal and external trauma reminders, which can result in increased behavioral flexibility and life satisfaction (Walser \& Hayes, 2006).

While emotion dysregulation has been linked with cognitive fusion and PTSD (e.g., Gillanders et al., 2014; O’Bryan, McLeish, Kraemer, \& Fleming, 2015), no studies have investigated which facets of emotion dysregulation mediate the fusion-PTSD link. One facet of emotion dysregulation that may explain the link between fusion and PTSD is emotional nonacceptance. Defusing from thoughts facilitates experiencing thoughts and feelings as external to the self (Greco, Lambert, \& Baer, 2008). This impersonalized perception of internal experiences may increase the willingness to accept and experience unpleasant internal states (Kishita, Muto, Ohtsuki, \& Barnes-Holmes, 2014). Contrarily, when cognitively fused, thoughts and feelings are personalized and interpreted as truth - increasing the likelihood of not accepting and avoiding unpleasant internal experiences and the external stimuli that trigger them. When trauma survivors are unwilling to experience trauma-related distress and avoid trauma-related stimuli that trigger distress, they inhibit cognitive-emotional processing (e.g., habituation) of traumas, perpetuating PTSD symptoms (Ehring \& Quack, 2010; Tull, Barrett, McMillan, \& Roemer, 2007; Weiss, Tull, Lavender, \& Gratz, 2013). However, if people are willing to accept their distressing emotional experiences following traumatic events, cognitive-emotional processing of 
the event will occur, which inhibits PTSD's development and maintenance. Thus, it may be that fusion's deleterious affect on acceptance explains its link with PTSD.

A second facet of emotion dysregulation that may explain the link between cognitive fusion and PTSD is emotional non-clarity. Researchers (e.g., Naragon-Gainey \& Demarree, 2017) have suggested that practices that provide cognitive distance from internal events-like defusion or decentering - are directly or indirectly associated with activation of the observer perspective, in which people gain a detached awareness of internal experiences. The distance that accompanies defused states facilitates clarity about both the emotions that are experienced and the situations that precipitated them (Boden \& Berenbaum, 2011).

Lack of emotional clarity has been consistently associated with PTSD (e.g., Ehring \& Quack, 2010; Tull et al., 2007). Within socialcognitive theory, people who understand how they feel and why they feel that way can form linear narratives explaining their distress. Simply having coherent explanations for the causes of difficult feelings has been associated with reduced mental health and physiological concerns (Pennebaker, Mayne, \& Francis, 1997). Coherent narratives reduce maladaptive self-reflective appraisals of distress (Pennebaker \& Seagal, 1999) —in other words — distress about distress (e.g., "I shouldn’t feel this way”). Neuroscientific evidence suggests that simply applying labels to emotions can disrupt cascading amygdala responses, such as anxiety and fear (Lieberman et al., 2007) and has been inserted into exposure therapies to more effectively treat PTSD (e.g., Foa, Hembree, \& Rothbaum, 2007). Emotional clarity also enables the accurate targeting of coping strategies by allowing people to select appropriate coping strategies based on accurate emotional information (Linehan, 2015). For example, in a study of veterans with PTSD, the use of cognitive reappraisal was only helpful in 
reducing PTSD for those who were high in emotional clarity (Boden, Bonn-Miller, Kashdan, Alvarez, \& Gross, 2012).

\subsection{Cognitive fusion and goal dysregulation}

When people are cognitively fused, behaviors are restricted due to over-identification with inhibiting thoughts (e.g., "I can’t do that”; Gillanders et al., 2014) and less able to accomplish their goals when they are distressed (i.e., goal dysregulated) (Paulus, Vanwoerden, Norton, \& Sharp, 2016). Laboratory studies have demonstrated that cognitive defusion interventions have led to more flexible behavioral responses and the ability to accomplish goals in the face of distress (Hooper \& McHugh, 2013; Levin, Hildebrandt, Lillis, \& Hayes, 2012; Ritzert, Forsyth, Berghoff, Barnes-holmes, \& Nicholson, 2015). As with the association between fusion and PTSD, there are empirical and theoretical reasons to hypothesize that emotional nonacceptance and emotional non-clarity mediate the association between fusion and goal dysregulation. Drawing from the evidence linking emotional non-acceptance and distress avoidance with the inability to accomplish desired tasks (e.g., Gerhart, Heath, Fitzgerald, \& Hoerger, 2013; Gratz, Rosenthal, Tull, Lejuez, \& Gunderson, 2006), it may be that nonacceptance impedes the ability to accomplish goals by encouraging escape behaviors. Additionally, being emotionally unclear may be overwhelming and make it difficult to understand and navigate the challenges that occur when pursuing goals (Hayes, 2002; Walser \& Hayes, 2006). Further, the emotional clarity that accompanies defusion may enhance the ability to identify and focus on long-term goals instead of becoming distracted by transient thoughts and feelings (Hayes, 2003). While there is substantial evidence for the link between fusion and goal dysregulation, there are few empirical investigations of the mediators of this association. 
Goal dysregulation has been consistently linked with PTSD symptoms (Ehring \& Quack, 2010; Tull et al., 2007; Weiss, Tull, Dixon- Gordon, \& Gratz, 2009). However, how emotional non-acceptance relates to the link between PTSD and goal dysregulation remains unclear. Some have argued that distress (e.g., PTSD symptoms) mediates the link between non-acceptance and behavioral responding; an unwillingness to experience distress results in greater distress, and distress reduces behavioral flexibility (Gerhart et al., 2013). This is consistent with behavioral explanations of the association between non-acceptance and PTSD (e.g., Ehring \& Quack, 2010; Tull et al., 2007; Weiss et al., 2013): Non-acceptance predicts PTSD, and PTSD-related distress impairs the ability to pursue goals. Others have suggested that acceptance buffers (i.e., moderates) the impact of distress on behavioral flexibility. In a study of statistics anxiety, willingness to experience anxiety buffered the effect anxiety had on statistics exam performance (Sandoz, Butcher, \& Protti, 2017). Further, in two studies of PTSD, avoidance exacerbated PTSD's link with impulsive behaviors (Bordieri, Tull, McDermott, \& Gratz, 2014; Gratz \& Tull, 2012). Findings from these studies support the moderating role of non-acceptance: Accepting distress facilitates goal attainment and non-acceptance inhibits it.

\subsection{Current study}

The purpose of our study was to develop and evaluate a model in which emotion dysregulation—specifically, emotional non-acceptance and emotional non-clarity—mediated the association between cognitive fusion and post-trauma functioning (e.g., PTSD symptoms, goal dysregulation, life satisfaction) among veterans with military-related trauma. The proposed model is shown in Fig. 1.

While our model has several embedded hypotheses, based on the reviewed empirical evidence and theory, our primary hypotheses were that (1) emotional non-acceptance and 
emotional non-clarity would mediate the association between cognitive fusion and PTSD symptoms; (2) emotional non-acceptance, emotional non-clarity, and PTSD symptoms would mediate the association between cognitive fusion and goal dysregulation; (3) PTSD and goal dysregulation would mediate the association between cognitive fusion and life satisfaction; and (4) emotional non-acceptance would moderate the association between PTSD symptoms and goal dysregulation.

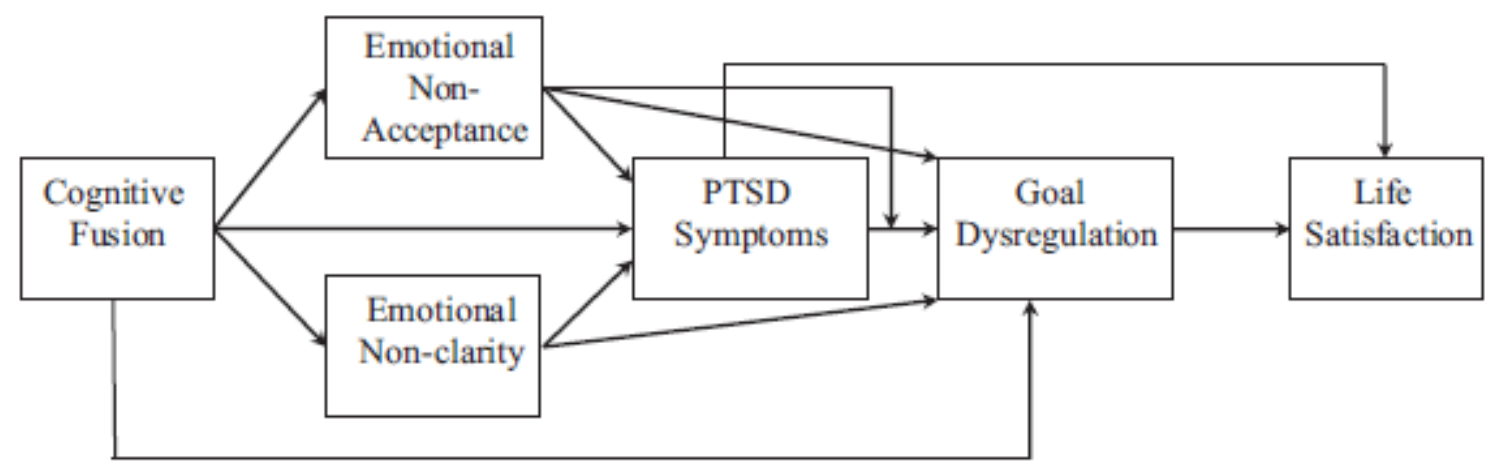

Fig. 1. Proposed path model (Model 1) delineating the indirect influence of cognitive fusion on PTSD symptoms and life satisfaction.

\section{Method}

\subsection{Participants and procedure}

Participants were 149 Canadian veterans enrolled in a post-service transition program for veterans struggling with emotional and interpersonal difficulties. All participants had experienced a traumatic life event while in the military. Data for the present study were collected prior to the program beginning by trained research assistants and was approved by the appropriate research-ethics board. Veterans ranged in age from 23 to 67 years ( $M=45.55$, $\mathrm{SD}=10.49)$ and were mostly men (89.9\%). The majority were married (62.8\%) followed by divorced (22.8\%), and single (12.8\%); the majority had children (69.1\%) and were heterosexual 
(92.0\%). Most veterans were Caucasian/White (92.0\%), Aboriginal (6.0\%), or East Asian

(2.0\%). Regarding branch of service, the majority served in the Army (63.8\%), Navy (11.4\%), Air Force (8.7\%), or multiple branches (16.1\%). Most participants had some college or more (71.1\%) and had annual household incomes of $\$ 60,000$ or more (62.0\%). The index traumatic events reported were military combat (65.1\%), motor vehicle accident (11.9\%), sexual assault (3.3\%), physical assault (11.1\%), childhood assault (6.5\%), and other (2.0\%). Based on empirically derived clinical cut-off scores on the PTSD assessment (Bovin et al., 2016), 79.3\% of the sample qualified for a DSM-5 PTSD diagnosis.

\subsection{Measures}

\subsubsection{Cognitive Fusion Questionnaire (CFQ)}

The CFQ (Gillanders et al., 2014) is a seven-item self-report measure of cognitive fusion (e.g., "I get so caught up in my thoughts that I am unable to do the things that I most want to do”). Respondents indicated how frequently they experienced cognitive fusion from 1 (never true) to 7 (always true). We derived total scores by summing item responses, with higher scores indicating greater cognitive fusion. The CFQ has been correlated—in theoretically consistent directions — with measures of mindfulness, psychological inflexibility, and the pursuit of valued goals. Psychometric support for the CFQ has been reported from clinical and non-clinical samples. Coefficient alpha in the present sample was .91.

\subsubsection{Difficulties with Emotion Regulation Scale (DERS)}

The DERS (Gratz \& Roemer, 2004) is a 36-item self-report measure of emotion dysregulation. Presently, we used three of the DERS subscales: non-acceptance of negative emotions (e.g., "When I’m upset, I become angry at myself for feeling that way”), lack of 
emotional clarity (e.g., "I have difficulty making sense out of my feelings”), and difficulty engaging in goal directed behaviors when distressed (e.g., "When I'm upset, I have difficulty getting work done”). The DERS has been correlated with measures of emotion-related behavior and psychopathology in theoretically consistent directions. Respondents indicated how frequently they experienced emotion dysregulation from 1 (almost never) to 5 (almost always). We derived subscale scores by summing item responses, with higher scores indicating greater emotion dysregulation. Coefficient alphas in the present sample were .89 for emotional nonacceptance (six items), .79 for emotional non-clarity (five items), and .86 for goal dysregulation (five items).

\subsubsection{Posttraumatic Stress Disorder Check List-5 (PCL-5)}

The PCL-5 (Weathers et al., 2013) is a 20-item self-report measure of DSM-5 PTSD symptom severity. Each PCL-5 item corresponds to a DSM- 5 PTSD symptom. Respondents indicated the extent to which they had been bothered by each PTSD symptom over the past month from 0 (not at all) to 4 (extremely). Respondents were instructed to indicate PTSD symptoms that were associated with their index traumatic event. Scores were summed and higher scores indicated greater PTSD symptom severity. A recent systematic review of PTSD assessments indicated that the PCL is one of two gold-standard self-report PTSD symptom measures (Spoont et al., 2015). Coefficient alpha in the present sample was .93.

\subsubsection{Satisfaction with Life Scale (SWLS)}

The SWLS (Diener, Emmons, Larsen, \& Griffin, 1985) is a five-item self-report measure of global life satisfaction. Respondents indicated how much they agreed with statements indicating life satisfaction (e.g., "I am satisfied with my life”) from 1 (strongly disagree) to 7 
(strongly agree). Scores were summed and higher scores indicated greater life satisfaction. The SWLS has been negatively correlated with measures of distress and negative affect and positively correlated with measures of desirable characteristics such as health, marital status, and subjective well-being (Pavot \& Diener, 1993). Coefficient alpha in the present sample was .87.

\subsubsection{The Traumatic Life Even Questionnaire (TLEQ)}

The TLEQ (Kubany et al., 2000) is a self-report measure of 22 potentially traumatic events. Respondents can also indicate traumatic events other than the 22 events explicitly assessed. At the end of the measure, respondents indicated their index traumatic event: the traumatic event that was causing them the most distress at that moment. Several indices of validity have been reported including a strong association with a structured interview of traumatic life events. We used the TLEQ to assess the index traumatic events reported in Section 2.1.

\subsection{Data analytic plan}

We used Mplus 7.11 (Muthén \& Muthén, 2012) to estimate path models, which included direct effects, indirect effects (i.e., mediation), and overall model fit. To ensure accurate tests of indirect effects, we used maximum likelihood estimation with bias-corrected bootstrapping with 10,000 random sample replacements. Bootstrapping facilitated the sampling distribution of the indirect effects being normally distributed. Statistically significant indirect effects were indicated by the $95 \%$ confidence intervals not including (i.e., straddling) a zero. We assessed model fit using standard cut-offs for several fit indices: Root mean square error of approximation (RMSEA) less than .05 (Browne \& Cudeck, 1993), the standardized root mean square residual less than .08 (Hu \& Bentler, 1999), as well as the comparative fit index (CFI) and Tucker-Lewis 
index (TLI) greater than .95. We also reported the chisquare value - a statistically significant chi-square indicates poor fit. It is important to note that chi-square is sensitive to sample size and model complexity, overly indicating significance (Tabachnick \& Fidell, 2013). Descriptive and frequency statistics as well as preliminary analyses were conducted using SPSS version 22 while path modelling was conducted using Mplus version 7.11.

\section{Results}

\subsection{Preliminary analyses}

The total scores of the primary study variables were screened for skewness and kurtosis via visual inspection of univariate histograms. We also evaluated skewness and kurtosis statistics using the $\mathrm{z}$ distribution (Tabachnick \& Fidell, 2013). All of the variables appeared normally distributed and $\mathrm{z}<1.96$. Further, there were no multivariate outliers observed when bivariate scatterplots were inspected. Bivariate correlations, means, and standard deviations are presented in Table 1. All of the primary variables were significantly correlated with each other in the expected directions. Neither the number of traumatic event types or the time since index events were significant in any of the models ( $p>.20$ ); thus, were not included in the models. All reported parameter estimates were standardized to facilitate ease of interpretation. 


\section{Table 1}

Bivariate correlations and descriptive statistics for primary variables.

\begin{tabular}{lllllll}
\hline Variable & 1 & 2 & 3 & 4 & 5 & 6 \\
\hline 1. Cognitive fusion & - & & & & & \\
2. Non-acceptance & $.66^{* *}$ & - & & & & \\
3. Emotional non-clarity & $.50^{* *}$ & $.38^{* *}$ & - & & & \\
4. Goal dysregulation & $.63^{* *}$ & $.51^{* *}$ & $.42^{* *}$ & - & & \\
5. PTSD symptoms & $.74^{* *}$ & $.52^{* *}$ & $.57^{* *}$ & $.61^{* *}$ & - & \\
6. Satisfaction with Life & $-.38^{* *}$ & $-.33^{* *}$ & $-.39^{* *}$ & $-.47^{* *}$ & $-.51^{* *}$ & - \\
$M$ & 33.32 & 18.54 & 15.38 & 18.83 & 47.49 & 15.69 \\
SD & 8.71 & 6.33 & 4.01 & 4.47 & 16.36 & 7.34 \\
Alpha & .91 & .89 & .79 & .86 & .93 & .87 \\
\hline
\end{tabular}

Note: PTSD $=$ posttraumatic stress disorder.

${ }^{* *} p<.01$.

\subsection{Path model}

We first tested the proposed model (see Fig. 1). Model 1 had a good fit to the data, $\chi^{2}(8$, $\mathrm{N}=149)=14.47, p=.070, \mathrm{CFI}=0.983, \mathrm{TLI}=.958, \mathrm{RMSEA}=.074$. SRMR $=.041$. However, the following direct paths were not statistically significant: Non-acceptance to PTSD and non-clarity to goal dysregulation. Therefore, to examine a more parsimonious model (Byrne, 2011), we tested a trimmed model with the non-significant paths constrained to zero (Model 2). Model 2 had a good fit to the data $\chi^{2}(10, \mathrm{~N}=149)=14.90, p=.136$, CFI $=.987$, TLI $=.974$, RMSEA $=.057$, $\mathrm{SRMR}=.042$. The $\chi^{2}$ difference between Model 2 and Model 1 was not significant, $\Delta \chi^{2}(2)=0.43$, $p>.25$, indicating that restraining the nonsignificant paths to zero did not significantly reduce the model fit (see Fig. 2). Thus, Model 2 was an improvement over Model 1 because all of the direct paths were statistically significant and the model was not a worse fit to the data. Model 2 explained $43 \%$ of the variance in non-acceptance, $25 \%$ of the variance in non-clarity, $60 \%$ of the 
variance in PTSD symptoms, $48 \%$ of the variance in goal dysregulation, and $29 \%$ of the variance in life satisfaction.

\subsection{Alternative models}

Next, we tested several alternative models to examine if any of them provided better fits to the data. We first calculated a nested model in which fusion, non-acceptance, and non-clarity were directly associated with life satisfaction (Model 3). Within Model 2, these constructs were only associated with life satisfaction through PTSD symptoms and goal dysregulation; however, they may have been directly related. The $\chi^{2}$ difference between Model 2 and Model 3 was not significant, $\Delta \chi^{2}(3)=2.78, \mathrm{p}>.25$, indicating that Model 3 did not provide a better fit to the data than Model 2. Further, none of the additional paths drawn in Model 3 were significant $(p>.10$ in all cases). We then tested a fourth model in which we interchanged PTSD and goal dysregulation (Model 4). It may have been that an inability to pursue goals when distressed was associated with increased behavioral avoidance and impeded PTSD symptom reduction. The RMSEA for Model 4 fell below the threshold indicating good fit and the chi-square test was statistically significant, $\chi^{2}(10, \mathrm{~N}=149)=46.214, \mathrm{p}<.001, \mathrm{CFI}=.906, \mathrm{TLI}=.811$, RMSEA $=.156$, SRMR $=.068$. Finally, we tested a fifth model in which we interchanged goal dysregulation and life satisfaction (Model 5). It may have been that satisfaction facilitated pursuing goals rather than the inverse. Again, this model did not fit the data as well as Model 2, $\chi^{2}(10, N=149)=39.685, p$ $<.001, \mathrm{CFI}=.923, \mathrm{TLI}=.845, \mathrm{RMSEA}=.141, \mathrm{SRMR}=.070$. 


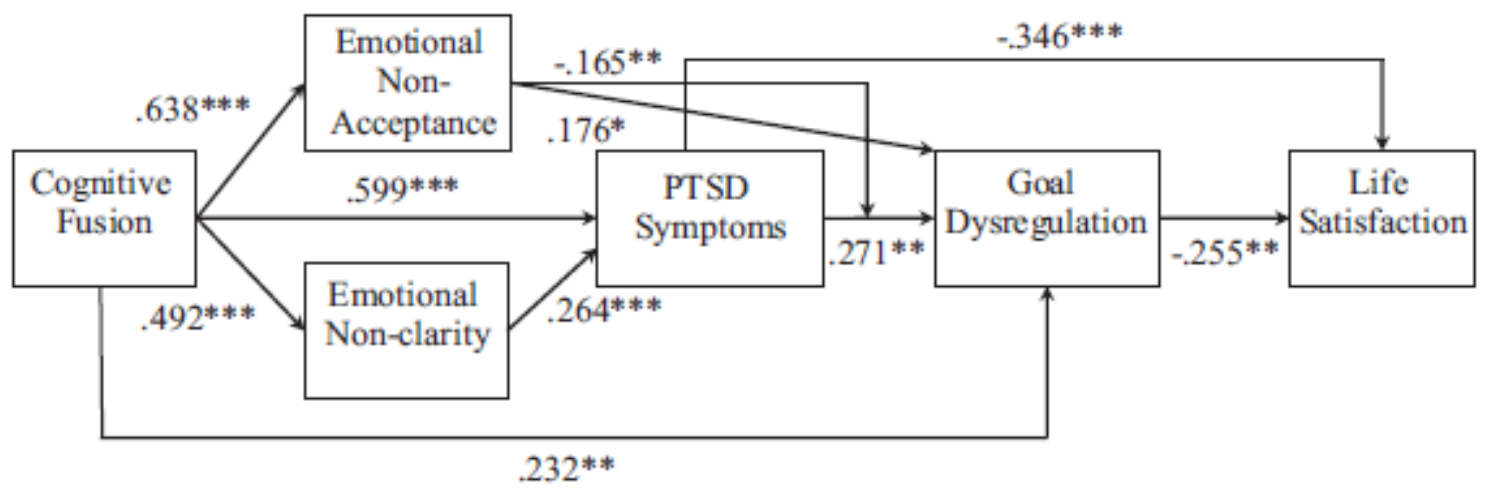

Fig. 2. Final path model (Model 2) with standardized coefficients. ${ }^{* * *} p<.001$, ${ }^{* *} p<.01,{ }^{*} p<.05$.

\subsection{Indirect effects}

To evaluate the potential mediating paths of the final model (i.e., Model 2), we examined the bias-corrected bootstrapped confidence intervals of the indirect effects. Confidence intervals that did not include (i.e., straddle) zero were considered statistically significant $(p<.05)$. All possible indirect effects through statistically significant direct effects are presented in Table 2.

Partially supporting our first hypothesis, the association between cognitive fusion and PTSD symptoms was mediated by emotional nonclarity ( $B=.13,95 \%$ CI $[.07, .19])$; however, not by emotional nonacceptance $(B=.03,95 \%$ CI $[-.07, .12])$. Partially supporting our second hypothesis, the link between cognitive fusion and goal dysregulation was mediated by emotional non-acceptance $(B=.11$ 95\% CI $[.01, .21])$ and PTSD symptoms ( $B=.16,95 \%$ CI $[.06, .27])$; however, not by emotional non-clarity $(B=.11,95 \% \mathrm{CI}[-.06, .09])$. It is worth noting that the two-step path of non-clarity to PTSD mediated the association between fusion and goal dysregulation ( $B=.04,95 \%$ CI $[.01, .06])$ indicating that while non-clarity alone did not mediate this association, non-clarity's association with PTSD did. Partially supporting our third hypothesis, the link between cognitive fusion and life satisfaction was mediated by PTSD 
symptoms ( $B=-.21,95 \% \mathrm{CI}[-.32,-.10])$; however, not by goal dysregulation $(B=-.06,95 \% \mathrm{CI}$ $[-.13, .01])$

\subsection{Interaction}

Supporting our fourth hypothesis, emotional non-acceptance significantly moderated the association between PTSD symptoms and goal dysregulation while controlling for the direct effects of cognitive fusion, emotional non-acceptance, and PTSD symptoms - the interaction term accounted for an additional 3\% of the variance in goal dysregulation above and beyond the direct effects. Follow-up simple slopes analysis revealed that the association between PTSD symptoms and goal dysregulation became stronger as non-acceptance increased from low $(-1$ $S D: B=.106,95 \%$ CI $[-.003, .215], p=.335)$ to the mean $(B=.271,95 \%$ CI $[.208, .334], p<.001)$ to high (+1SD: $B=.436,95 \%$ CI [.327, .545], $p<.001)$ (see Fig. 3).

\section{Discussion}

\subsection{General discussion}

Presently, we developed a theory-based model of the associations between cognitive fusion, emotion dysregulation (i.e., emotional nonacceptance and emotional non-clarity), and post-trauma functioning in a trauma-exposed veteran sample. In our initial model, we hypothesized that non-acceptance and non-clarity would mediate both the fusion- PTSD and fusion-goal dysregulation associations. Consistent with prior cognitive distancing research (e.g., Naragon-Gainey \& Demarree, 2017), these primary relations were supported. Defusion was associated with both non-clarity and non-acceptance. However, our subsequent hypotheses were only partially supported, as only non-clarity mediated the association with PTSD symptoms, and only non-acceptance mediated the association with goal dysregulation. The non-clarity and 
nonacceptance paths connecting fusion to PTSD, goal dysregulation, and life satisfaction are used to explain how these variables may be related, and are discussed below.

Table 2

Standardized coefficients of bootstrap tests of indirect effects for the final path model (Model 2).

\begin{tabular}{ll}
\hline Independent and mediator variables & Standardized coefficients \\
\hline Fusion $\rightarrow$ Non-clarity $\rightarrow$ PTSD & .130 \\
Fusion $\rightarrow$ PTSD $\rightarrow$ Goal dysregulation & .163 \\
Fusion $\rightarrow$ Non-clarity $\rightarrow$ PTSD $\rightarrow$ Goal dysregulation & .035 \\
Fusion $\rightarrow$ Non-acceptance $\rightarrow$ Goal dysregulation & .112 \\
Fusion $\rightarrow$ PTSD $\rightarrow$ Goal dysregulation $\rightarrow$ Life Satisfaction & -.042 \\
Fusion $\rightarrow$ Non-clarity $\rightarrow$ PTSD $\rightarrow$ Goal dysregulation $\rightarrow$ Life Satisfaction & $95 \%$ CI \\
Fusion $\rightarrow$ Goal dysregulation $\rightarrow$ Life Satisfaction & -.009 \\
Fusion $\rightarrow$ Non-acceptance $\rightarrow$ Goal dysregulation $\rightarrow$ Life Satisfaction & $.007, .063$ \\
Fusion $\rightarrow$ PTSD $\rightarrow$ Life Satisfaction & -.059 \\
Fusion $\rightarrow$ Non-clarity $\rightarrow$ PTSD $\rightarrow$ Life satisfaction & -.029 \\
Clarity $\rightarrow$ PTSD $\rightarrow$ Goal dysregulation & -.208 \\
Clarity $\rightarrow$ PTSD $\rightarrow$ Life satisfaction & -.045 \\
Clarity $\rightarrow$ PTSD $\rightarrow$ Goal dysregulation $\rightarrow$ Life satisfaction & .072 \\
Non-acceptance $\rightarrow$ Goal dysregulation $\rightarrow$ Life satisfaction & -.091 \\
PTSD $\rightarrow$ Goal dysregulation $\rightarrow$ Life satisfaction & -.002 \\
\hline
\end{tabular}

Note: Fusion = cognitive fusion; non-clarity = emotional non-clarity; non-acceptance = emotional non-acceptance; PTSD = posttraumatic stress disorder symptoms; bolded confidence intervals do not include a zero, indicating a significant indirect effect.

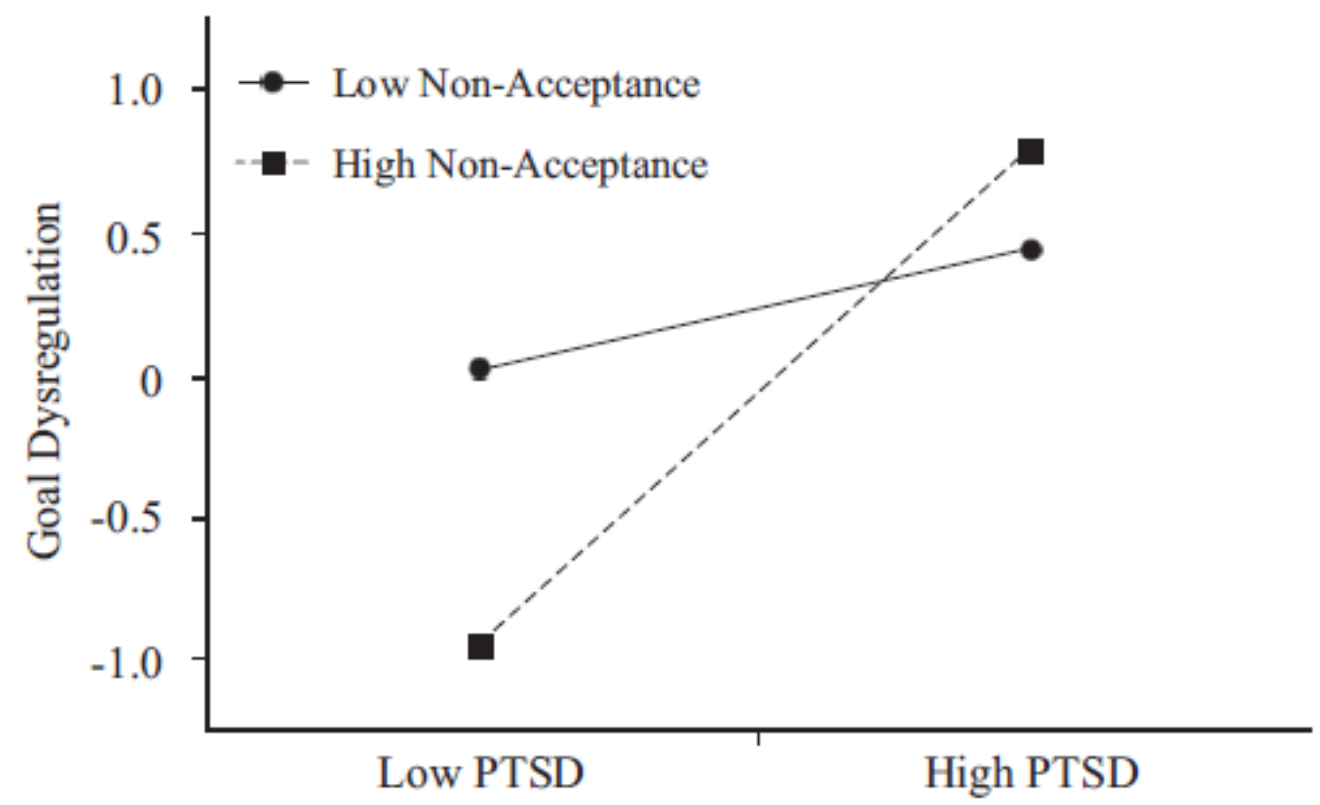

Fig. 3. Simple slopes of the association between PTSD symptoms and goal dysregulation for those with low $(-1 S D)$ and high $(+1 S D)$ emotional non-acceptance.

Our findings are consistent with others indicating that cognitive fusion is associated with reduced emotional clarity (e.g., Naragon- Gainey \& Demarree, 2017). Conversely, people who 
are defused may be better able to identify the presence, causes, and consequences of aversive internal events, increasing emotional clarity (Walser \& Westrup, 2007). Consistent with our model, the emotion labeling that occurs when having emotional clarity has been linked with reduced distress associated with unpleasant emotions (e.g., Pennebaker \& Seagal, 1999) as well as implementing appropriate coping behaviors or self-regulatory strategies (e.g., Boden et al., 2012). Further, in our model, PTSD symptoms mediated the relation between emotional nonclarity and goal dysregulation while non-clarity did not directly contribute to goal dysregulation. Based on these findings, it may be that emotional nonclarity is related to difficulty pursuing desired goals only inasmuch as non-clarity produces or maintains PTSD symptoms. Based on the current model, increased clarity enables PTSD symptom management, which can create the context to pursue goals. Overall, this path may be summarized: People who are defused have greater emotional clarity, which is associated with reduced PTSD symptoms and effective goal pursuits.

Cognitive fusion was also associated with impaired emotional acceptance. When people are cognitively fused and do not accept unpleasant emotions, their emotions inhibit their ability to accomplish their goals (Hayes, 2004). Defusion enables people to alter their relationships with internal events, such that unpleasant emotions, like the anxiety associated with PTSD, are experienced without requiring behavioral efforts to control or eliminate them (e.g., Hooper \& Mchugh, 2013; Levin et al., 2012). Those who are more able to accept aversive internal experiences are more able to pursue their goals and values without re-prioritizing their behaviors to avoid or escape unwanted internal events. Within this pathway, defusion is associated with altered relationships with their internal events and the willingness to experience them. Regardless of the type and intensity of emotions (what is experienced), the ability to accept 
emotions without enacting control strategies alters how emotions function. Consistent with acceptancebased theory (e.g., Hayes, 2004), non-acceptance was not associated with reduced PTSD symptoms, but instead to the ramifications of those symptoms on the ability to pursue goals. Overall, this path may be summarized: People who are defused are more accepting of their emotions, which is related to flexible goal pursuits regardless of PTSD symptom severity.

\subsection{Practical implications}

Generally, our findings support the assertion that cognitive fusion is an important phenomenon for practitioners to target as it has substantial direct and indirect associations with psychological distress, the ability to accomplish goals, and life satisfaction. Further, researchers and practitioners should continue to collaborate to develop, evaluate, and disseminate interventions that facilitate defusion. More specifically, our study indicates a nuanced perspective of how cognitive fusion may function that could be helpful for practitioners as they consider their clinical goals. If the goal is to reduce PTSD symptoms, practitioners should consider defusion techniques that facilitate emotional clarity. However, if the clinical aim is to facilitate goal pursuit, practitioners should consider defusion techniques that enable emotional acceptance. While these differences are subtle, practitioners conceptualizing their clinical aims and interventions using this framework could facilitate more targeted explanations to clients, interventions, and processing, which could enhance client buy-in and therapeutic potency. Drawing from common interventions used to facilitate defusion (Hayes, 2005): the Describing Thoughts and Feelings exercise in which clients take distressing issues and give them physical descriptors (e.g., What color is it? What texture does it have?) may most strongly impact emotional clarity. However, the Don’t Think about Your Thoughts exercise in which clients 
attempt to suppress distressing thoughts with the therapeutic goal of recognizing that suppression only increases the thoughts' impact may most strongly impact acceptance.

\subsection{Limitations \& future directions}

This study had several limitations that should be considered. First, the data were cross sectional, reducing our ability to optimally test for causal effects. Future research should use longitudinal and experimental methods to better understand the causal nature of the associations we investigated in veteran samples. Second, all of the data were self-reported. Using observational and other methods would help reduce some of the biases native to self-report data. Also, our sample lacked in certain diversities. Specifically, it was mostly Caucasian and mostly male. More diverse samples would facilitate testing if the associations observed in the current study generalize to other populations. Finally, researchers have conceptualized emotion regulation in many ways. Presently, we used a conceptualization of emotion regulation that was theoretically consistent with the model being investigated. Future research that uses other conceptualizations of emotion regulation to examine the association between cognitive fusion and post-trauma functioning are important for better understanding these phenomena. 


\section{References}

Bardeen, J. R., \& Fergus, T. A. (2016). The interactive effect of cognitive fusion and experiential avoidance on anxiety, depression, stress and posttraumatic stress symptoms. Journal of Contextual Behavioral Science, 5(1), 1-6. http://dx.doi.org/10.1016/j.jcbs.2016.02.002.

Boden, M. T., \& Berenbaum, H. (2011). What you are feeling and why: Two distinct types of emotional clarity. Personality and Individual Differences, 51(5), 652-656. http://dx.doi.org/10.1016/j.paid.2011.06.009.

Boden, M. T., Bonn-Miller, M. O., Kashdan, T. B., Alvarez, J., \& Gross, J. J. (2012). The interactive effects of emotional clarity and cognitive reappraisal in Posttraumatic Stress Disorder. Journal of Anxiety Disorders, 26(1), 233-238. http://dx.doi.org/10.1016/j.janxdis.2011.11.007.

Bordieri, M. J., Tull, M. T., McDermott, M. J., \& Gratz, K. L. (2014). The moderating role of experiential avoidance in the relationship between posttraumatic stress disorder symptom severity and cannabis dependence. Journal of Contextual Behavioral Science, 3(4), 273278. http://dx.doi.org/10.1016/j.jcbs.2014.08.005.

Bovin, M. J., Marx, B. P., Weathers, F. W., Gallagher, M. W., Rodriguez, P., Schnurr, P. P., \& Keane, T. M. (2016). Psychometric properties of the PTSD checklist for diagnostic and statistical manual of mental disorders-fifth edition (PCL-5) in veterans. Psychological Assessment, 28, 1379-1391. http://dx.doi.org/10.1037/pas0000254.

Browne, M. W., \& Cudeck, R. (1993). Alternative ways of assessing model fit. In K. A. Bollen, \& J. S. Long (Eds.). Testing structural equation models (pp. 136-162). Newbury Park, CA: Sage.

Byrne, B. M. (2011). Structural equation modeling with Mplus: Basic concepts, applications, and programming. New York, NY: Routledge.

Cahill, S. P., Rothbaum, B. O., Resick, P. A., \& Follette, V. M. (2009). Cognitive-behavioral therapy for adults. In E. B. Foa, T. M. Keane, M. J. Friedman, \& J. D. Cohen (Eds.). Effective treatments for PTSD (pp. 139-222). (2nd ed.). New York, NY: The Guilford Press. 
Diener, E., Emmons, R. A., Larsen, R. J., \& Griffin, S. (1985). The Satisfaction with Life Scale. Journal of Personality Assessment, 49(1), 71-75. http://dx.doi.org/10.1207/s15327752jpa4901_13.

Ehring, T., \& Quack, D. (2010). Emotion regulation difficulties in trauma survivors: The role of trauma type and PTSD symptom severity. Behavior Therapy, 41(4), 587-598. http://dx.doi.org/10.1016/j.beth.2010.04.004.

Foa, E. B., Ehlers, A., Clark, D. M., Tolin, D. F., \& Orsillo, S. M. (1999). The Posttraumatic Cognitions Inventory (PTCI): Development and validation. Psychological Assessment, 11(3), 303-314. http://dx.doi.org/10.1037/1040-3590.11.3.303.

Foa, E. B., Hembree, E. A., \& Rothbaum, B. O. (2007). Prolonged exposure therapy for PTSD: Emotional processing of traumatic experiences. New York, NY: Oxford University Press.

Gerhart, J. I., Heath, N. M., Fitzgerald, C., \& Hoerger, M. (2013). Direct and indirect associations between experiential avoidance and reduced delay of gratification. Journal of Contextual Behavioral Science, 2(1-2), 9-14. http://dx.doi.org/10.1016/j.jcbs.2013.02.001.

Gillanders, D. T., Bolderston, H., Bond, F. W., Dempster, M., Flaxman, P. E., Campbell, L., \& Remington, B. (2014). The development and initial validation of the Cognitive Fusion Questionnaire. Behavior Therapy, 45(1), 83-101. http://dx.doi.org/10.1016/j.beth.2013.09.001.

Gratz, K. L., \& Roemer, L. (2004). Multidimensional assessment of emotion regulation and dysregulation: Development, factor structure, and initial validation of the Difficulties in Emotion Regulation Scale. Journal of Psychopathology and Behavioral Assessment, 26(1), 41-54. http://dx.doi.org/10.1023/B:JOBA.0000007455.08539.94.

Gratz, K. L., Rosenthal, M. Z., Tull, M. T., Lejuez, C. W., \& Gunderson, J. G. (2006). An experimental investigation of emotion dysregulation in borderline personality disorder. Journal of Abnormal Psychology, 115(4), 850-855. http://dx.doi.org/10.1016/j.comppsych.2015.10.004. 
Gratz, K. L., \& Tull, M. T. (2012). Exploring the relationship between posttraumatic stress disorder and deliberate self-harm: The moderating roles of borderline and avoidant personality disorders. Psychiatry Research, 199(1), 19-23. http://dx.doi.org/10.1016/j.psychres.2012.03.025.

Greco, L. A., Lambert, W., \& Baer, R. A. (2008). Psychological inflexibility in childhood and adolescence: Development and evaluation of the Avoidance and Fusion Questionnaire for Youth. Psychological Assessment, 20(2), 93-102. http://dx.doi.org/10.1037/10403590.20.2.93.

Hayes, S. C. (2002). Buddhism and acceptance and commitment therapy. Cognitive and Behavioral Practice, 9(1), 58-66. http://dx.doi.org/10.1016/S1077-7229(02)80041-4.

Hayes, S. C. (2004). Acceptance and commitment therapy, relational frame theory, and the third wave of behavioral and cognitive therapies. Behavior Therapy, 35, 639-665. http://dx.doi.org/10.1016/S0005-7894(04)80013-80013.

Hayes, S. C. (2005). Get out of your mind \& into your life: The new acceptance and commitment therapy. Oakland, CA: New Harbinger Publications, Inc.

Hooper, N., \& McHugh, L. (2013). Cognitive defusion versus thought distraction in the mitigation of learned helplessness. The Psychological Record, 63(1), 209-218.

Hu, L., \& Bentler, P. M. (1999). Cutoff criteria for fit indexes in covariance structure analysis: Conventional criteria versus new alternatives. Structural Equation Modeling: A Multidisciplinary Journal, 6(1), 1-55. http://dx.doi.org/10.1080/10705519909540118.

Kishita, N., Muto, T., Ohtsuki, T., \& Barnes-Holmes, D. (2014). Measuring the effect of cognitive defusion using the Implicit Relational Assessment Procedure: An experimental analysis with a highly socially anxious sample. Journal of Contextual Behavioral Science, 3(1), 8-15. http://dx.doi.org/10.1016/j.jcbs.2013.12.001.

Kubany, E. S., Haynes, S. N., Leisen, M. B., Owens, J. A., Kaplan, A. S., Watson, S. B., \& Burns, K. (2000). Development and preliminary validation of a brief broad-spectrum measure of trauma exposure: The Traumatic Life Events Questionnaire. Psychological Assessment, 12(2), 210-224. http://dx.doi.org/10.1037/1040-3590.12.2.210. 
Levin, M. E., Hildebrandt, M. J., Lillis, J., \& Hayes, S. C. (2012). The impact of treatment components suggested by the psychological flexibility model: A meta-analysis of laboratory-based component studies. Behavior Therapy, 43(4), 741-756. http://dx.doi.org/10.1016/j.beth.2012.05.003.

Lieberman, M. D., Eisenberger, N. I., Crockett, M. J., Tom, S. M., Pfeifer, J. H., \& Way, B. M. (2007). Putting feelings into words: Affect labeling disrupts amygdala activity in response to affective stimuli. Psychological Science, 18(5), 421-428. http://dx.doi.org/10.1111/j.1467-9280.2007.01916.x.

Linehan, M. M. (2015). DBT skills training manual (2nd ed.). New York, NY: Guilford Press.

Muthén, L. K., \& Muthén, B. O. (2012). Mplus user's guide (Seventh). Los Angeles, CA: Muthén \& Muthén.

Naragon-Gainey, K., \& Demarree, K. G. (2017). Structure and validity of measures of decentering and defusion. Psychological Assessment, 29(7), 935-954. http://dx.doi.org/10.1037/pas0000405.

O’Bryan, E. M., McLeish, A. C., Kraemer, K. M., \& Fleming, J. B. (2015). Emotion regulation difficulties and posttraumatic stress disorder symptom cluster severity among traumaexposed college students. Psychological Trauma: Theory, Research, Practice, and Policy, 7(2), 131-137. http://dx.doi.org/10.1037/a0037764.

Paulus, D. J., Vanwoerden, S., Norton, P. J., \& Sharp, C. (2016). Emotion dysregulation, psychological inflexibility, and shame as explanatory factors between neuroticism and depression. Journal of Affective Disorders, 190, 376-385. http://dx.doi.org/10.1016/j.jad.2015.10.014.

Pavot, W., \& Diener, E. (1993). Review of the Satisfaction With Life Scale. Psychological Assessment, 5(2), 164-172. http://dx.doi.org/10.1037//1040-3590.5.2.164.

Pennebaker, J. W., Mayne, T. J., \& Francis, M. E. (1997). Linguistic predictors of adaptive bereavement. Journal of Personality and Social Psychology, 72(4), 863-871. http://dx.doi.org/10.1037/0022-3514.72.4.863. 
Pennebaker, J. W., \& Seagal, J. D. (1999). Forming a story: The health benefits of narrative. Journal of Clinical Psychology, 55(10), 1243-1254. http://dx.doi.org/10.1002/(SICI)1097-4679(199910)55.

Ritzert, T. R., Forsyth, J. P., Berghoff, C. R., Barnes-holmes, D., \& Nicholson, E. (2015). The impact of a cognitive defusion intervention on behavioral and psychological flexibility: An experimental evaluation in a spider fearful non-clinical sample. Journal of Contextual Behavioral Science, 4(2), 112-120. http://dx.doi.org/10.1016/j.jcbs.2015.04.001.

Sandoz, E. K., Butcher, G., \& Protti, T. A. (2017). A preliminary examination of willingness and importance as moderators of the relationship between statistics anxiety and performance. Journal of Contextual Behavioral Science, 6(1), 47-52. http://dx.doi.org/10.1016/j.jcbs.2017.02.002.

Spoont, M. R., Williams, J. W., Kehle-Forbes, S., Nieuwsma, J. A., Mann-Wrobel, M. C., \& Gross, R. (2015). Does this patient have posttraumatic stress disorder? Rational clinical examination systematic review. JAMA, 314(5), 501-510. http://dx.doi.org/10.1001/jama.2015.7877.

Surís, A., Lind, L., Kashner, T. M., \& Borman, P. D. (2007). Mental health, quality of life, and health functioning in women veterans: Differential outcomes associated with military and civilian sexual assault. Journal of Interpersonal Violence, 22(2), 179-197. http://dx.doi.org/10.1177/0886260506295347.

Tabachnick, B. G., \& Fidell, L. S. (2013). Using multivariate statistics (6th ed.). Boston, MA: Pearson.

Tull, M. T., Barrett, H. M., McMillan, E. S., \& Roemer, L. (2007). A preliminary investigation of the relationship between emotion regulation difficulties and posttraumatic stress symptoms. Behavior Therapy, 38(3), 303-313. http://dx.doi.org/10.1016/j.beth.2006.10.001.

Twohig, M. P. (2009). Acceptance and commitment therapy for treatment-resistant posttraumatic stress disorder: A case study. Cognitive and Behavioral Practice, 16(3), 243-252. http://dx.doi.org/10.1016/j.cbpra.2008.10.002. 
Vogt, D. S., King, D. W., King, L. A., Saverese, V. W., \& Suvak,, M. K. (2004). War-zone exposure and long-term general life adjustment among Vietnam veterans: Findings from two perspectives. Journal of Applied Social Psychology, 34(9), 1797-1824. http://dx.doi.org/10.1111/j.1559-1816.2004.tb02586.x.

Walser, R. D., \& Hayes, S. C. (2006). Acceptance and commitment therapy in the treatment of posttraumatic stress disorder. In V. C. Follette, \& J. I. Ruzek (Eds.). Cognitive behavioral therapies for trauma (pp. 146-172). (2nd ed.). New York, NY: The Guilford Press.

Walser, R. D., \& Westrup, D. (2007). Acceptance \& Commitment Therapy for the treatment of post-traumatic stress disorder \& trauma-related problems. Oakland, CA: New Harbinger Publications, Inc.

Weathers, F. W., Litz, B.T., Keane, T.M., Palmieri, P.A., Marx, B.P., \& Schnurr, P.P. (2013). The PTSD Checklist for DSM-5 (PCL-5). Scale available from the National Center for PTSD at 〈www.ptsd.va.gov〉.

Weiss, N. H., Tull, M. T., Dixon-Gordon, K. L., \& Gratz, K. L. (2009). Extending findings of a relation between posttraumatic stress disorder and emotion dysregulation among African American individuals: A preliminary examination of the moderating role of gender. Journal of Traumatic Stress Disorders \& Treatment, 27(5), 417-428. http://dx.doi.org/10.1055/s-0029-1237430.

Weiss, N. H., Tull, M. T., Lavender, J., \& Gratz, K. L. (2013). Role of emotion dysregulation in the relationship between childhood abuse and probable PTSD in a sample of substance abusers. Child Abuse and Neglect, 37(11), 944-954. http://dx.doi.org/10.1016/j.chiabu.2013.03.014. 